\title{
Tracking and Alignment of the LHCb detector
}

\author{
M. MARTINELLI* \\ Nikhef, \\ Science Park 105, Amsterdam, The Netherlands \\ *E-mail: maurizio.martinelli@nikhef.nl \\ www.nikhef.nl
}

\begin{abstract}
The LHCb experiment is designed to study B-decays at the LHC. This purpose is achieved by means of an outstanding track momentum resolution and excellent particle identification. The performance of the detector exceeds expectations, and the physics program of the collaboration is extended to the study of charm decays, QCD and electroweak physics. Both tracking and alignment play a key role for obtaining these results. The tracking efficiency in this highoccupancy environment has been measured during LHC Run I (2010-2012) to be larger than $95 \%$ over a wide range of momentum and pseudo-rapidity. The alignment of the detector is based on a Kalman filter fit of the tracks and provides an uncertainty on the momentum scale below $1 \times 10^{-4}$ and a momentum resolution of about $0.4 \%$. These results allowed one to achieve high-precision mass measurements of many charm and $B$ hadrons so far. Starting from Run II of the LHC, the running conditions will continue to push the performance of the detector to its limits. An upgrade of the detector will therefore take place in 2018 to extend its reach. A fast tracking will be a fundamental part of the upgraded detector as well. In light of this, the prospects for the recently proposed upgrade will be discussed.
\end{abstract}

Keywords: LHCb; LHCb Upgrade; Alignment; Tracking System.

\section{Tracking at $\mathrm{LHCb}$}

The $\mathrm{LHCb}$ detector is a single arm spectrometer designed for reconstructing $B$ mesons produced in $p p$ interactions and decaying in the forward region. ${ }^{1}$ Nevertheless, its excellent performance allowed the collaboration to extend the LHCb physics case to charm physics, and to produce electroweak and QCD measurements complementary to ATLAS and CMS in the high forward region. A few examples that show the potential of the detector are the precise determination of $B_{s}^{0}$ oscillations, ${ }^{2}$ that shows the excellent decay time resolution of the detector (about $50 \mathrm{fs}$ ), the first evidence for the $B_{s}^{0} \rightarrow \mu^{+} \mu^{-}$decay, ${ }^{3}$ allowed by the high reconstruction efficiency and 
the good mass resolution, and the most precise search for $C P$ violation in $D^{0}-\bar{D}^{0}$ mixing, ${ }^{4}$ possible thanks to the unprecedented recorded yield of charm decays.

The tracking system of $\mathrm{LHCb}$ is divided in a silicon detector close to the interaction region, a dipole magnet, and tracking stations before and after the magnet. The magnetic field provides momentum estimate, and its polarity is inverted regularly during data taking for reducing the systematics from charge asymmetry in the detector. The silicon detector close to the interaction region (VELO) is used both for vertexing and tracking. Between this detector and the magnet there are four layers of silicon strip detectors, the Tracker Turicensis (TT). The tracking system behind the magnet is divided in two parts: a small silicon detector covering the highest particle flux region, the Inner Tracker (IT), and a gaseous straw tube detector, the Outer Tracker (OT), covering the lower flux region. Particle identification is done by two ring-imaging Cherenkov detectors, RICH1 and RICH2, devoted to low- and high-momentum tracks identification, respectively. These detectors are placed between VELO and TT (RICH1), and after the tracking stations (RICH2). Behind RICH2, the first muon station, electromagnetic and hadronic calorimeters, and four other muon stations are placed.

The information of all the tracking stations is used to achieve the best reconstruction efficiency and momentum resolution keeping the rate of fake tracks and clones under control. The tracking reconstruction can be split in two parts: pattern recognition and fit. The pattern recognition identifies patterns of hits that are likely to originate from the same track. The fit uses these hits as input to provide the best estimate of the track.

Many pattern algorithms are used at LHCb for reconstructing charged particles. They are defined as follows: Velo tracks are reconstructed from hits in the VELO; Long tracks are made from the Velo tracks adding hits in the tracking stations (IT/OT); the remaining hits in the tracking stations are used for reconstructing $T$ tracks; Upstream tracks are made with VELO tracks and hits in the TT; Downstream tracks are made with $\mathrm{T}$ tracks and remaining hits in the TT. The Long tracks are most used for reconstructing $B$ and $D$ decay products, while Downstream are used for reconstructing long-lived particles such as $K_{S}^{0}$ and $\Lambda$.

After the pattern recognition a track fit algorithm is run, based on a Kalman filter. ${ }^{5}$ A Kalman filter is used since it guarantees a fast estimation of the track parameters with good accuracy, and it is easy to include effects from external sources, such as multiple scattering and energy loss, directly 
into the fit.

The tracking efficiency has been measured for the data recorded during Run I of the LHC (2010-2012). The measurement is based on a tag-andprobe method, where $J / \psi \rightarrow \mu^{+} \mu^{-}$decays are used. ${ }^{6}$ Three efficiencies are calculated depending on the information used by the reconstruction algorithm of the probe track, $\varepsilon_{\text {Long }}, \varepsilon_{V e l o}$, and $\varepsilon_{T}$. For evaluating $\varepsilon_{\text {Long }}$, an algorithm that makes use of TT and muon stations hits only is used. Similarly $\varepsilon_{V \text { elo }}$ probes tracks with the Downstream algorithm, and $\varepsilon_{T}$ with a VELO-muon algorithm. By construction $\varepsilon_{\text {Long }} \approx \varepsilon_{V e l o} \varepsilon_{T}$, therefore the three efficiencies are used to measure the same Long efficiency in two different ways, giving consistent results. This method allows one to measure the efficiency of the different tracking algorithms on data, which can then be compared to a Monte Carlo sample. The resulting efficiency has been found to be larger than $95 \%$ over a wide range of track momentum and pseudo-rapidity, in good agreement to the Monte Carlo. This result is particularly important for reducing the systematic error in cross-section and branching-ratio measurements. Using this technique, a systematic error of $2 \%$ per track is estimated.

\section{Alignment of the $\mathrm{LHCb}$ detector}

The alignment at $\mathrm{LHCb}$ is strictly connected to tracking. There are two algorithms available for aligning the detector: a method based on Millipede, ${ }^{7}$ and a track fit-based alignment. ${ }^{8}$ The former uses a simplified track model, and can align only one sub-detector at once, but converges within one iteration. The latter includes the alignment parameters in the track fit model and finds the optimal alignment parameters by minimizing the $\chi^{2}$ calculated on all the tracks considered as input. Furthermore, it accounts for multiple scattering and energy loss effects. The two algorithms produce consistent results and have been both tested on the first data taken in 2010. Since the track fit-based has the advantage of being able to align many sub-detectors at once, it is the one used by default.

The alignment of the LHCb detector consists of four steps, corresponding to the detectors that are aligned: VELO, tracker, RICH mirrors, and muon stations. Each step can be run independently of the other one, but performing them in the presented sequence improves the results.

The VELO alignment ${ }^{9,10}$ takes as input tracks that have produced many hits $(>36)$ or that have traversed the overlap region for more than 16 modules in this sub-detector. In addition, tracks from the primary vertex are used and the primary vertex itself is added as a constraint to the alignment 
fit. The z-scale is fixed by constraining the first and last module of the detector to their survey position, known with an accuracy of $10 \mu \mathrm{m}$. A few validation tests are performed once the alignment has converged. A $4 \mu \mathrm{m}$ residual misalignment is estimated from the hit residuals. The VELO is made of two retractable halves that are open during beam injection and close around the collision point with stable beams. Its alignment stability is tested by reconstructing the primary vertex position with the tracks from the left and right half of the VELO separately, and the distance between the two reconstructed points is considered. The displacement is within 5(2) $\mu \mathrm{m}$ in $\mathrm{x}(\mathrm{y})$. One of the main achievement of the alignment is the impact parameter resolution, that is $12 \mu \mathrm{m}$ for high momentum particles.

The alignment of the tracking system uses a specific selection of tracks produced by two-body decays of well known resonances $\left(D^{0}, J / \psi, Z^{0}\right)$, and a mass constraint on the vertex of the two tracks is applied to fix the momentum scale. ${ }^{11}$ A few more tracks are also selected, especially if they have crossed overlap regions in the detectors. The sub-detectors aligned are the TT, the IT, and the OT. The alignment constants are updated every magnet polarity switch or after the technical stops to account for any change in detectors condition that may have happened during the time. A detailed study of the alignment constants over time has shown that they present small fluctuations of about $100 \mu \mathrm{m}$ and $1 \mathrm{mrad}$ for the translational and rotational degrees of freedom, respectively. Even if small, these fluctuations affect the mass measurements, and need to be corrected for obtaining the required precision. A comparison is done between the data reprocessed with preliminary 2010 alignment and the latest 2012 alignment. Among the effects of tracker alignment one gets an improved track quality, for example the average track $\chi^{2}$ probability is doubled, and an observed curvature bias is corrected. The improvement on mass resolution is noticeable, the $\Upsilon(1 S)$ mass resolution is $44 \mathrm{MeV} / c^{2}$ with the latest detector alignment, while it was $86 \mathrm{MeV} / c^{2}$ with the preliminary alignment. A residual momentum scale is still found and produces an effect on the mass measurement of many resonances of about $3 \times 10^{-4}$.

This effect is too large for precise measurements of particle masses. A momentum calibration is therefore applied at a later stage. Kaons from well reconstructed $B^{+} \rightarrow J / \psi K^{+}$decays are used to correct the momentum scale in bins of $t_{x}=p_{x} / p_{z}$ and $t_{y}=p_{y} / p_{z}$. The output of this calibration is tested on various resonances over a wide mass range. The momentum scale effect is reduced to less than $1 \times 10^{-4}$, and the momentum resolution to $0.4 \%$ (i.e. a relative $7 \%$ improvement). This allowed one to provide the 
best mass measurements of the $D^{+}, D_{s}^{+}$, and $B$ mesons, and the $\Lambda_{b}^{0}, \Omega_{b}^{-}$, $\Sigma_{b}^{-}$baryons so far. ${ }^{12-16}$

The muon stations alignment uses muons from $J / \psi \rightarrow \mu^{+} \mu^{-}$as input with at least 4 hits in these stations. Since the offline reconstruction is marginally affected by small misalignments in the muon stations, it is performed a few times per year. ${ }^{17}$

Misalignments in the RICH mirrors are solved by applying corrections to the distribution of two variables: the difference between the observed Cherenkov angle and the one estimated from the momentum of the incoming track, $\Delta \theta_{C}$, and the polar angle measured from the vector that defines the distance of the point where the track hit the detector and the reconstructed center of the Cherenkov ring $(\phi)$. In case of mirror misalignment the distribution of $\Delta \theta_{C}$ vs. $\phi$ has a sinusoidal behavior: $\Delta \theta_{C}=\theta_{x} \cos \phi+\theta_{y} \cos \phi$. This is corrected by fitting this distribution to extract $\theta_{x}$ and $\theta_{y} \cdot{ }^{18}$

\section{Future Prospects}

The collaboration is now preparing for Run II of the LHC, during which the detector will face running conditions that will push its performance to the limit. The $b \bar{b}$ cross section will double when the center-of-mass energy of the $p p$ collisions increments from $8 \mathrm{TeV}$ to $14 \mathrm{TeV}$. Furthermore, the bunch spacing will reduce from $50 \mathrm{~ns}$ to $25 \mathrm{~ns}$. These conditions are just an appetizer for what will happen after 2018, when LHCb is planning to improve the luminosity by a factor 5 . For this to happen a large upgrade of the detector is needed. ${ }^{19,20}$ The upgrade is triggered by the need of updating the detector readout to $40 \mathrm{MHz}$ from the actual $1 \mathrm{MHz}$. Many sub-detectors therefore need to be redesigned and for some of them a new technology has been chosen. Among the tracking detectors, the VELO is changing from silicon strips to pixels, the TT is being redesigned to enlarge coverage in the high pseudo rapidity region, and for the tracker there are a couple of options under study. Either the IT surface will be enlarged and the OT consistently reduced, or all tracking stations will be substituted by a full scintillating fiber detector. Tests of the tracking algorithms have been crucial to determine the best hardware solution to be used in the upgrade. All the algorithms have performed well with few modifications in the simulations of the new detector. The global detector alignment is not yet investigated due to the missing technology choice on the tracking stations, but the prospects are positive given the good performance of the tracking algorithms. 


\section{Summary}

In conclusion, the $\mathrm{LHCb}$ experiment is producing outstanding results in flavor physics. Tracking and alignment are crucial, providing a very high tracking efficiency (over 95\%), an excellent impact parameter resolution for high momentum tracks $(12 \mu \mathrm{m})$, a decay time resolution of about $50 \mathrm{fs}$, a momentum scale around $1 \times 10^{-4}$ and a momentum resolution below $0.5 \%$. In the future, the $\mathrm{LHCb}$ detector will face a major upgrade. Most of the tracking algorithms have been successfully applied in simulations to the new detector layout, showing excellent performances with minor modifications.

\section{References}

1. A. A. Alves, Jr. et al. [LHCb Collaboration], JINST 3, S08005 (2008).

2. R. Aaij et al. [LHCb Collaboration], New J. Phys. 15, 053021 (2013) [arXiv:1304.4741 [hep-ex]].

3. R. Aaij et al. [LHCb Collaboration], Phys. Rev. Lett. 111, 101805 (2013) [arXiv:1307.5024 [hep-ex]].

4. R. Aaij et al. [LHCb Collaboration], arXiv:1309.6534 [hep-ex].

5. R. Fruhwirth, Nucl. Instrum. Meth. A 262, 444 (1987).

6. A. Jaeger et al.,LHCb-PUB-2011-025.

7. V. Blobel, Nucl. Instrum. Meth. A 566, 5 (2006).

8. W. Hulsbergen, Nucl. Instrum. Meth. A 600, 471 (2009) [arXiv:0810.2241 [physics.ins-det]].

9. S. Viret et al.,Nucl. Instrum. Meth. A 596, 157 (2008) [arXiv:0807.5067 [physics.ins-det]].

10. M. Gersabeck et al.,Nucl. Instrum. Meth. A 596, 164 (2008) [arXiv:0807.5069 [physics.ins-det]].

11. J. Amoraal et al.,Nucl. Instrum. Meth. A 712, 48 (2013) [arXiv:1207.4756 [physics.ins-det]].

12. R. Aaij et al. [LHCb Collaboration], Phys. Rev. Lett. 109, 232001 (2012) [arXiv:1209.5634 [hep-ex]].

13. R. Aaij et al. [LHCb Collaboration], Phys. Rev. D 87, 112012 (2013) [arXiv:1304.4530 [hep-ex]].

14. R. Aaij et al. [LHCb Collaboration], JHEP 1306, 065 (2013) [arXiv:1304.6865 [hep-ex]].

15. R. Aaij et al. [LHCb Collaboration], Phys. Lett. B 708, 241 (2012) [arXiv:1112.4896 [hep-ex]].

16. R. Aaij et al. [LHCb Collaboration], Phys. Rev. Lett. 110, 182001 (2013) [arXiv:1302.1072 [hep-ex]].

17. A. A. Alves et al.,JINST 8, P02022 (2013) [arXiv:1211.1346 [physics.ins-det]].

18. M. Adinolfi et al.,Eur. Phys. J. C 73, 2431 (2013) [arXiv:1211.6759 [physics.ins-det]].

19. R. Aaij et al. [LHCb collaboration], CERN-LHCC-2011-001 (2011).

20. R. Aaij et al. [LHCb collaboration], CERN-LHCC-2012-007 (2012). 